\title{
Cushing's Disease in a 7-year-boy due to corticotroph cell hyperplasia.
}

\section{Introduction}

* Cushing's disease is the most common cause of endogenous Cushing's syndrome in children after the age of $5^{1}$

* It accounts for $70-85 \%$ of all paediatric Cushing's syndrome.

* In childhood it is male predominant, which equilibrates at puberty, and becomes female predominant as adults

* The majority of Cushing's disease are caused by an ACTHsecreting pituitary corticotroph adenoma.

* Ectopic ACTH is extremely rare.

* Corticotroph cell hyperplasia has only been convincingly shown in two previous cases of paediatric Cushings disease ${ }^{2}$

\section{Clinical Presentation \& Examination}

7- year old presented with a
ten-month history of obesity,
hirsutism and growth
retardation.
He was a term baby and
reached all appropriate
developmental milestones.
No specific drugs had been
prescribed previously.

\section{$\checkmark$ Moon face \\ $\checkmark$ Facial plethora \\ $\checkmark$ Buffalo hump \\ $\checkmark$ Central obesity \\ $\checkmark$ Hirsutism \\ $\checkmark$ Striae}

Hgt: 2.5SD below agemean

Wgt: 98 $^{\text {th }}$ percentile

\section{Diagnostic Imaging}

\section{* Noncontrast CT Adrenals.} \& MRI pituitary: unremarkable
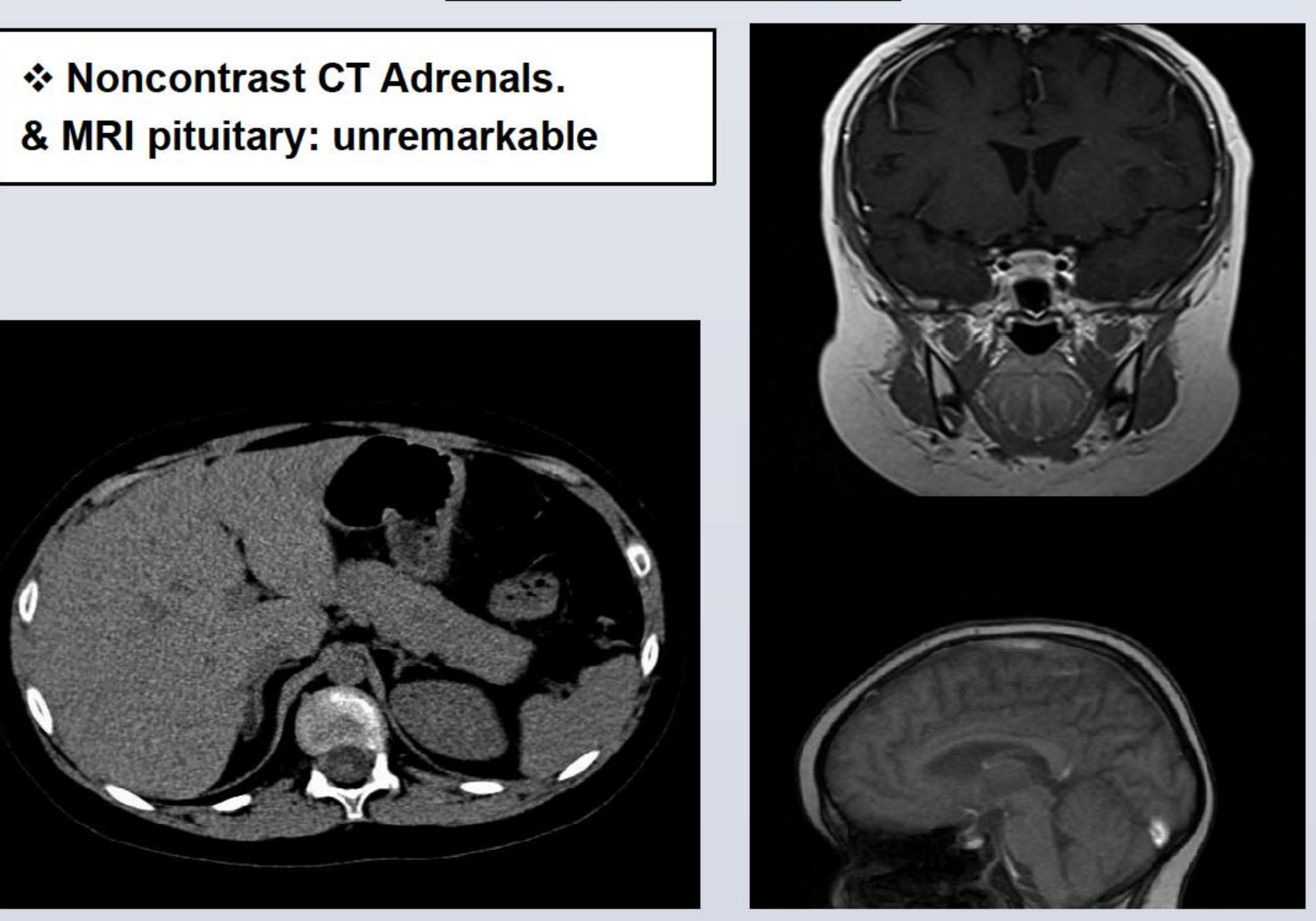

\section{Management}

* Underwent Transphenoidal Surgery 3-months post IPSS confirmation of ACTH dependant Cushings syndrome.

* Abnormal tissue was resected from the left side of the pituitary.

* Histopathology revealed no adenoma but intense immunostaining for ACTH consistent with corticotroph hyperplasia.

* On the fourth day post-operation, am cortisol was $39 \mathrm{nmol} / \mathrm{L}$

* Three months post TSS, he remained hypocortisolaemic on hydrocortisone with significant clinical improvement.

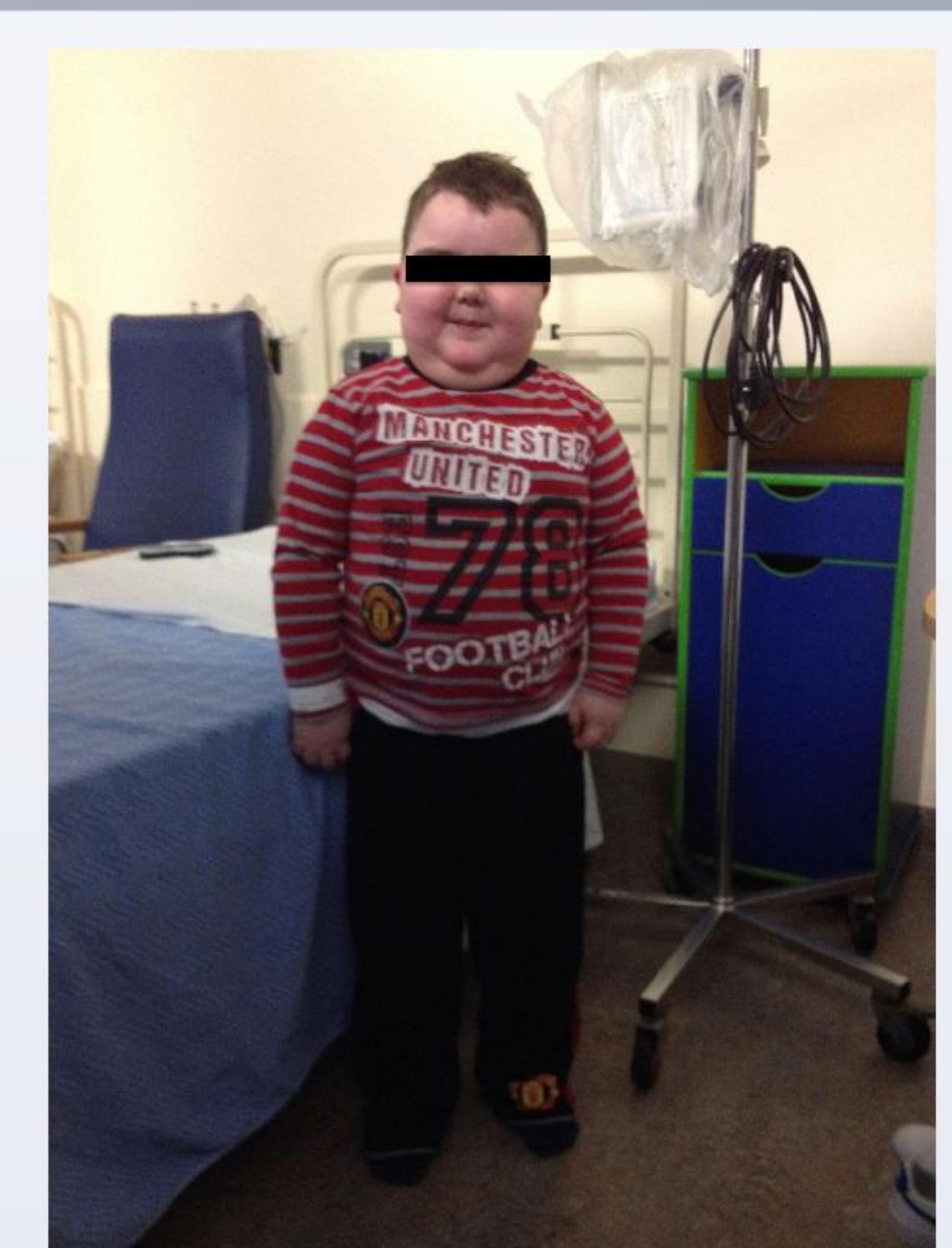

Fig 1.At presentation Oct'13.

\section{Initial investigations}

$\begin{array}{ll}\text { 8am Cortisol } & 1159 \mathrm{nmol} / \mathrm{L}(185 \\ & 624 \mathrm{nmol} / \mathrm{L}) \\ & 488.0 \mathrm{\rho g} / \mathrm{ml} \\ \text { (8am)ACTH } & (10-60 \mathrm{pg} / \mathrm{ml}) \\ & 13.9 \mathrm{nmol} / \mathrm{L} \\ \text { Midnight Salivary Cortisol } & (<2.6 \mathrm{nmol} / \mathrm{L}) \\ & 69.2 \mathrm{nmol} / \mathrm{L} \\ \text { Midnight Salivary Cortisone } & (<18 \mathrm{nmol} / \mathrm{L}) \\ & 1010 \mathrm{~mL} / 24 \mathrm{hrs} \\ \text { Urine Total Volume } & 232 \mathrm{nmol} / 24 \mathrm{hrs}\end{array}$

Table 1 \& 2 Endocrine testing at presentation

\section{Inferior Petrosal Sinus Sampling}

\begin{tabular}{|l|l|l|l|l|l|}
\hline $\begin{array}{l}\text { ACTH Sample } \\
(\rho g / \mathrm{ml})\end{array}$ & Peripheral & Left IPS & $\begin{array}{l}\text { Right } \\
\text { IPS }\end{array}$ & $\begin{array}{l}\text { Highest } \\
\text { IPS : peripheral } \\
\text { ratio }\end{array}$ & $\begin{array}{l}\text { Lateralization } \\
\text { ratio }\end{array}$ \\
\hline Basal & 142 & 159 & 142 & $159 / 142=1.1$ & \\
\hline Post CRH & & & & & \\
\hline$\underline{3 \mathrm{~min}}$ & 676 & 630 & 768 & & \\
\hline $10 \mathrm{~min}$ & 488 & $>1950$ & 560 & $>1950 / 488=>4$ & $>1950 / 560=>3.5$ \\
\hline $15 \mathrm{~min}$ & & 1176 & 602 & & \\
\hline
\end{tabular}

\section{Conclusion}

* Early diagnosis of paediatric Cushings disease remains a challenge.

* Definitive cure can be achieved by transphenoidal pituitary surgery but success rates vary from $45 \%$ to $78 \%$ in report series ${ }^{3}$.

* This case ilustrates that pediatric Cushing's disease may be caused,albeit, rarely by corticotroph hyperplasia.

* The natural history of this entity is unknown,hence careful follow up is necessary.

\section{References}

Storr HL, Chan LF, Grossman AB, Savage MO. Paediatric Cushing's Disease: Epidemiology, Investigation and Therapeutic Advances. Trends Endocrinol Metab. 2007

2. Mustila T,Keskinen P,Terho M,Huovinen S, Saha MT.Pediatric Cushing's disease due to pituitary hyperplasia. J Pediatr Endocrinol Metab. 2011;24(3-4):191-2

3. Storr HL, Alexandraki KI, Martin L, Isidori AM, Kaltsas G, Monson JP, et al. Comparisons in the epidemiology, diagnostic features and cure rate by transsphenoidal therapy between paediatric and adult Cushing's disease. Eur J Endocrinol. 2011 